\title{
Otimização de Parâmetros Operacionais do Processo de Soldagem FCAW para Aplicação em Revestimento Duro
}

\author{
Hebert dos Santos Cavalcante ${ }^{1}$, Francisco Machado de Assis Bentes Neto², Carlos Alberto Mendes da Mota² (D), Douglas Neves Garcia ${ }^{1}$ (D) \\ ${ }^{1}$ Universidade Federal do Pará - UFPA, Faculdade de Engenharia Mecânica, Tucuruí, PA, Brasil. \\ 2 Universidade Federal do Pará - UFPA, Faculdade de Engenharia Mecânica, Programa de Pós-graduação em Engenharia Mecânica, Belém, PA, Brasil.
}

Como citar: Cavalcante HS, Bentes Neto FMA, Mota CAM, Garcia DN. Otimização de parâmetros operacionais do processo de soldagem FCAW para aplicação em revestimento duro. Soldagem \& Inspeção. 2020;25:e2522. https://doi.org/10.1590/0104-9224/SI25.22

\begin{abstract}
Resumo: Neste trabalho foi investigada a influência de variáveis de soldagem sobre a geometria dos cordões de solda, depositados pelo processo FCAW, visando a aplicação de revestimentos duros. Chapas de aço carbono SAE 1020 foram utilizadas como substrato, e como metal de adição uma liga $\mathrm{FeCrC}+\mathrm{Nb}$, diâmetro 1,6 mm. Como variáveis de entrada foram analisadas a tensão (U), velocidade de alimentação de arame (Va) e velocidade de soldagem (Vs), mantendo constante os demais parâmetros utilizou-se um planejamento composto central (PCC) em três níveis. A otimização do modelo estatístico ocorreu através da Metodologia de Superfície de Respostas (MSR) e do método de otimização de respostas múltiplas (desirability). Os resultados revelaram modelo matemático estatisticamente significativo e preditivo para a resposta Reforço/Largura (R/L). Para a resposta Área reforço/Área fundida (Ar/Af), o modelo se caracterizou como estatisticamente significativo, não preditivo, necessitando de um melhor ajuste. A velocidade de alimentação foi a variável com a maior significância para as respostas R/L e Ar/Af, enquanto a tensão foi a menos significativa.
\end{abstract}

Palavras-chave: DOE; Metodologia superfície de resposta; Desirability; Arame FeCrC+Nb.

\section{Operational Parameters Optimization of FCAW Welding Process for Hardfacing Applications}

\begin{abstract}
This work assessed the influence of welding parameters over weld beads geometry, deposited by FCAW process, aiming for hardfacing applications. SAE 1020 steel plates were used as substrate and $1.6 \mathrm{~mm}$ FeCrC+Nb alloy wire as filler metal. The input parameters were voltage (U), wire feed speed (WFS) and travel speed (TS), maintaining the remaining parameters constant a central composite design (CCD) was used in three levels. The statistical model optimization was performed through Response Surface Methodology (RSM) and multiple response optimization methodology (desirability). The results revealed a statistically significant mathematical model and predictive for Reinforcement/Width (R/W) response. For Reinforcement Area/Penetration Area ( $\mathrm{Ra} / \mathrm{Pa})$ response, the model was characterized as statistically significant, non-predictive, requiring better fit. The wire feed speed was the most significant variable for $\mathrm{R} / \mathrm{W}$ and $\mathrm{Ra} / \mathrm{Pa}$ responses, while voltage was the less significant.
\end{abstract}

Key-words: DOE; Surface response methodology; Desirability; FeCrC+Nb wire.

\section{Introdução}

Nos diversos ramos industriais a perda de material por desgaste causa danos consideráveis em componentes e equipamentos. Os danos por abrasão é uma das principais causas de redução da vida útil de máquinas e equipamentos nas indústrias de mineração e agrícola [1-5]. A deposição de revestimento duro é uma das técnicas mais eficientes e econômicas empregadas para enfrentar o desgaste abrasivo severo de ferramentas agrícolas, componentes para equipamentos de mineração e revestimento de chute $[1,3,5,6]$. Nesta técnica, camadas superficiais duras e resistentes ao desgaste são depositadas na superfície de um substrato metálico macio por soldagem, com o objetivo de aumentar a resistência ao desgaste abrasivo sem perda significativa da tenacidade do substrato [7].

Vários processos de soldagem são concorrentes para aplicação em revestimentos de superfícies, sendo que a utilização da soldagem com arame tubular (FCAW- flux cored arc welding) reúne vantagens metalúrgicas à alta produtividade, boa qualidade e baixo custo [3,8]. No entanto, este processo de soldagem apresenta uma gama considerável de fatores operacionais de influência sobre a qualidade e as propriedades do metal de solda depositado, dentre os quais destacam-se a corrente, a tensão e a velocidade de soldagem, fluxo e distância bico de contato peça. 
Na soldagem de revestimento é importante controlar a geometria e a diluição dos cordões de solda. Pois, cordões com alta convexidade poderão apresentar falta de fusão entre passes, e uma elevada penetração (maior diluição) irá influenciar na resistência ao desgaste, já que as ligas bifásicas são dependentes da fração volumétrica, tamanho, morfologia e dureza da segunda fase [2,3,5,8]. De acordo com Fratari et al. [9], o índice de convexidade (IC) tem valores aceitáveis de qualidade quando próximo de $30 \%$. O IC é definido segundo a Equação 1.

$I C=\frac{R}{L} * 100 \%$

onde: R é o reforço e L é a largura do cordão de solda.

Devido ao grande número de fatores de influência do processo de soldagem sobre as soldas produzidas, uma alternativa que vem sendo utilizada por alguns pesquisadores [10-14] é a aplicação das técnicas de análise de experimentos DOE (Design of Experiments). Para Rowlands e Antony [15], o DOE é uma poderosa ferramenta utilizada na investigação dos efeitos causados pelos parâmetros de processo, os quais afetam significativamente as respostas de processos/produtos. Esta ferramenta quando associada com a metodologia de superfície de resposta (MSR ou RSM - Response Surface Methodology) possibilita conhecer o ponto ótimo do processo em questão, ou seja, quais devem ser os ajustes dos parâmetros de processo para se obter a melhor resposta.

Segundo Dowlatshahi [16], a utilização de ferramentas estatísticas (DOE) é possível reduzir o número de ensaios necessários para conhecer quais parâmetros produzem efeitos significativos nas respostas. Sendo assim, o DOE possibilita o estudo e análise de um grande número de fatores de maneira rápida e econômica. De acordo com Barros et al. [17], para eliminar a ocorrência de desvio estatístico nos resultados, isto é, para evitar que distorções atípicas sejam obrigatoriamente associadas a determinadas combinações de níveis, deve-se realizar os ensaios em ordem aleatória.

Dessa maneira, este estudo teve o objetivo de avaliar a influência das variáveis de entrada (tensão, velocidade de alimentação e velocidade de soldagem) sobre as características geométricas (relação reforço/largura e relação área do reforço/área fundida) e diluição de soldas de revestimento duro de uma liga FeCrC+Nb depositadas pelo processo FCAW, através da Metodologia de Superfície de Respostas e da técnica conhecida como "desirability, garantindo um cordão de solda com características desejáveis ao revestimento duro.

\section{Materiais e Métodos}

A soldagem pelo processo FCAW autoprotegido foi automatizada e realizada por uma fonte eletrônica tensão constante, em simples deposição sobre chapas de aço carbono SAE 1020, medindo $150 \times 50 \times 10 \mathrm{~mm}$. O metal de adição foi uma liga $\mathrm{FeCrC}+\mathrm{Nb}$ na forma de arame tubular com diâmetro de 1,6 mm. As composições químicas dos metais de adição e da chapa são apresentadas na Tabela 1 . As soldas foram depositadas na posição plana, empurrando com inclinação de $15^{\circ}$, e distância bico de contato peça (DBCP) de $35 \mathrm{~mm}$ para todas as condições experimentais estabelecidas.

Tabela 1. Composição química dos metais base e adição (\% em peso).

\begin{tabular}{ccccc}
\hline & $\mathbf{C}$ & $\mathbf{M n}$ & $\mathbf{C r}$ & $\mathbf{N b}$ \\
SAE 1020 & $0,18-0,23$ & $0,3-0,6$ & - & - \\
FeCrC+Nb & $5,0-6,0$ & $0,5-1,0$ & $18,0-22,0$ & $4,0-7,0$ \\
\hline
\end{tabular}

Fonte: KESTRA [18] e GERDAU [19]

\subsection{Planejamento experimental}

A influência dos fatores foi verificada através de um planejamento composto central $2^{3}$, contendo três fatores em dois níveis $\left(2^{k}=2^{3}=8\right)$, seis pontos axiais $(2 k=2.3=6)$, e dois pontos centrais $(n=2)$, totalizando 16 experimentos. Os experimentos foram realizados sem repetição e em ordem aleatória.

A escolha dos fatores e dos níveis foi baseada em estudos preliminares e pré-testes, sendo considerado para este sistema a influência de três variáveis de entrada: a tensão (U), a velocidade de alimentação do arame (Va) e a velocidade de soldagem $(V s)$, e tendo como variáveis de respostas específicas do processo as relações reforço/largura (R/L) e área reforço/área fundida (Ar/Af). A Tabela 2 apresenta os parâmetros de soldagem utilizados como variáveis de entrada e seus respectivos níveis.

As respostas de interesse na soldagem de revestimento duro são uma alta deposição e baixa diluição. Alta deposição é representada pela área de reforço (Ar), enquanto a diluição apresenta uma relação direta com a área fundida (Af). Neste caso, é desejável maximizar a relação $\operatorname{Ar} / \operatorname{Af}[9,10]$. No entanto, devem-se considerar restrições quanto a convexidade do cordão, obtida através das dimensões lineares do reforço e da largura, assim pode-se esperar que quanto menor a relação R/L menor 
será a convexidade do cordão $[9,10,20]$. Dessa maneira, considerando a geometria do cordão de solda, desejam-se a maximização da relação Ar/Af e a minimização da relação R/L.

Tabela 2. Valores reais e codificados dos parâmetros.

\begin{tabular}{ccc}
\hline Parâmetros do processo & Unidade & Níveis dos parâmetros $\mathbf{- 1} \mathbf{0}+\mathbf{1}$ \\
Tensão & $\mathrm{V}$ & 283032 \\
Velocidade de soldagem & $\mathrm{cm} \cdot \mathrm{min}^{-1}$ & 304050 \\
Velocidade de alimentação & $\mathrm{m} \cdot \mathrm{min}^{-1}$ & 101214 \\
\hline
\end{tabular}

As Figuras 1a e 1b ilustram os procedimentos utilizados para a medição das características geométricas dos depósitos de solda. Observa-se pela Figura 1a que os cortes e medições foram realizadas em três pontos distintos ao longo do comprimento do cordão, com a finalidade de obter uma média das medições. Com a ajuda do software analisador de imagens ImageJ, as dimensões do cordão foram medidas obtendo-se a largura, a penetração, o reforço, a área do reforço e a área fundida da solda.

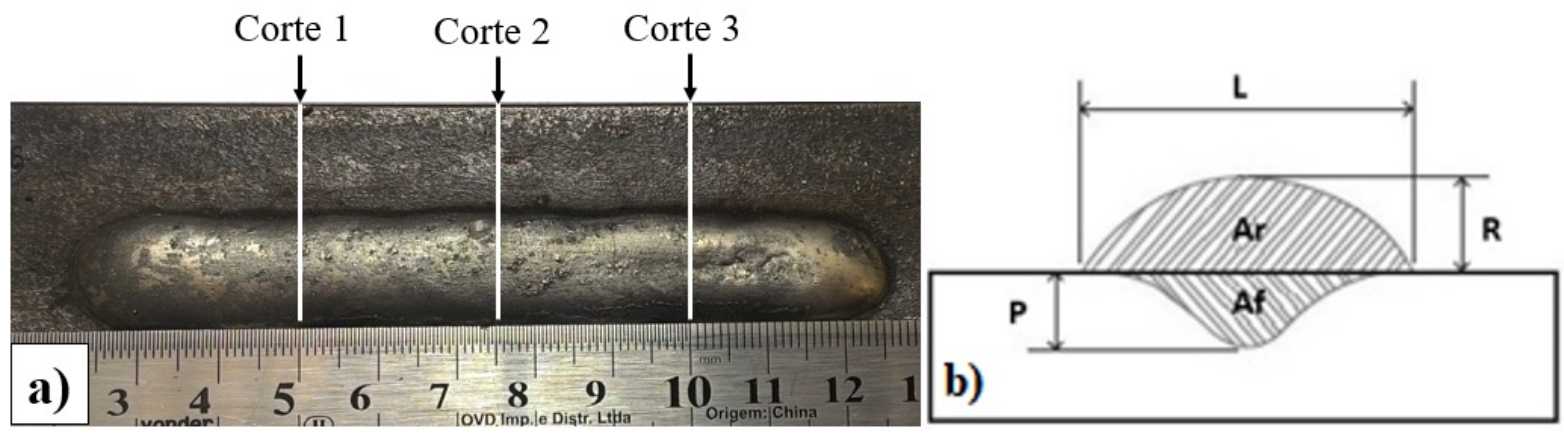

Figura 1. Cordão isolado. (a) sequência dos cortes no corpo de prova; (b) representação esquemática da seção transversal do cordão.

Os valores de diluição (D) dos cordões de soldas foram calculados através das medições das áreas Ar e Af (Figura 1b) utilizando o software Image J. A Equação 2 foi utilizada para determinar os níveis de diluição.

$D(\%)=\frac{A f}{A r+A f} * 100 \%$

\section{Resultados e Discussões}

A Tabela 3 apresenta os resultados das características geométricas dos cordões de solda, tendo como variáveis independentes a tensão $U(V)$, a velocidade de alimentação $V a\left(m \cdot \mathrm{min}^{-1}\right)$ e a velocidade de soldagem Vs $\left.\left(\mathrm{cm} . \mathrm{mim}^{-1}\right)\right)$. Pode-se observar que a relação área do reforço/área fundida (Ar/Af) variou de 1,77 a 4,11, enquanto a relação reforço/largura do cordão (R/L) variou de 24 a 37,32\%. Pode-se também observar que todos os cordões apresentaram reforço superior a 3,2 mm. O valor máximo da diluição deve ser controlado e não pode ser muito elevado, a fim de garantir a resistência ao desgaste do depósito [1-5]. Já uma penetração (diluição) mínima precisa ser alcançada de modo a garantir uma desejada resistência ao arrancamento da camada de revestimento [20].

Tabela 3. Matriz de planejamento composto central (PCC) e resultados obtidos.

\begin{tabular}{|c|c|c|c|c|c|c|c|c|c|c|c|c|}
\hline \multirow{2}{*}{ Teste } & \multicolumn{3}{|c|}{ Fatores de entrada } & \multicolumn{3}{|c|}{ Fatores de entrada } & \multicolumn{6}{|c|}{ Respostas (Fatores de saída) } \\
\hline & $\mathbf{U}$ & Va & Vs & $\mathbf{U}$ & Va & Vs & $\mathbf{L}$ & $\mathbf{R}$ & $R / L$ & Ar & Af & Ar/Af \\
\hline 1 & -1 & -1 & -1 & 28 & 10 & 30 & 11,58 & 4,30 & 37,12 & 45,79 & 11,14 & 4,11 \\
\hline 2 & -1 & -1 & 1 & 28 & 10 & 50 & 10,12 & 3,40 & 33,61 & 28,32 & 12,07 & 2,35 \\
\hline 3 & -1 & 1 & -1 & 28 & 14 & 30 & 15,28 & 4,78 & 31,28 & 71,41 & 26,20 & 2,73 \\
\hline 4 & -1 & 1 & 1 & 28 & 14 & 50 & 15,03 & 4,17 & 27,74 & 51,11 & 26,85 & 1,90 \\
\hline 5 & 1 & -1 & -1 & 32 & 10 & 30 & 13,97 & 3,77 & 26,99 & 58,41 & 19,12 & 3,05 \\
\hline 6 & 1 & -1 & 1 & 32 & 10 & 50 & 12,32 & 3,33 & 27,04 & 33,58 & 16,75 & 2,01 \\
\hline 7 & 1 & 1 & -1 & 32 & 14 & 30 & 16,17 & 4,55 & 28,14 & 66,87 & 24,00 & 2,79 \\
\hline
\end{tabular}




\begin{tabular}{|c|c|c|c|c|c|c|c|c|c|c|c|c|}
\hline \multirow{2}{*}{ Teste } & \multicolumn{3}{|c|}{ Fatores de entrada } & \multicolumn{3}{|c|}{ Fatores de entrada } & \multicolumn{6}{|c|}{ Respostas (Fatores de saída) } \\
\hline & $\mathbf{U}$ & Va & Vs & $\mathbf{U}$ & Va & Vs & $\mathbf{L}$ & $\mathbf{R}$ & $R / L$ & $\mathrm{Ar}$ & Af & Ar/Af \\
\hline 8 & 1 & 1 & 1 & 32 & 14 & 50 & 14,53 & 3,83 & 26,35 & 46,41 & 18,30 & 2,54 \\
\hline 9 & -1 & 0 & 0 & 28 & 12 & 40 & 14,58 & 3,80 & 26,06 & 42,04 & 19,00 & 2,21 \\
\hline 10 & 1 & 0 & 0 & 32 & 12 & 40 & 15,27 & 3,93 & 25,74 & 43,67 & 24,66 & 1,77 \\
\hline 11 & 0 & -1 & 0 & 30 & 10 & 40 & 11,07 & 4,13 & 37,32 & 32,19 & 10,19 & 3,16 \\
\hline 12 & 0 & 1 & 0 & 30 & 14 & 40 & 13,48 & 4,28 & 31,74 & 43,61 & 18,07 & 2,41 \\
\hline 13 & 0 & 0 & -1 & 30 & 12 & 30 & 16,37 & 4,30 & 26,27 & 55,54 & 28,20 & 1,97 \\
\hline 14 & 0 & 0 & 1 & 30 & 12 & 50 & 13,67 & 3,28 & 24,00 & 38,41 & 18,66 & 2,06 \\
\hline $15 \mathrm{C}$ & 0 & 0 & 0 & 30 & 12 & 40 & 14,20 & 4,08 & 28,73 & 43,76 & 17,17 & 2,55 \\
\hline $16 \mathrm{C}$ & 0 & 0 & 0 & 30 & 12 & 40 & 14,67 & 3,85 & 26,25 & 49,83 & 17,17 & 2,90 \\
\hline
\end{tabular}

As medições das respostas de interesse foram reunidas para a composição de uma matriz experimental, utilizada como fonte de dados para a estimação dos modelos matemáticos aplicados. Para a análise dos resultados foi utilizado um software Statistica através do Método dos Mínimos Quadrados Ordinários. Neste trabalho foi empregado um nível de significância $\rho<0,05$, para um intervalo de confiança de 0,95. As hipóteses formuladas são:

$\left(\mathrm{H}_{\circ}\right)$ Os fatores não produzem efeitos significativos;

$\left(\mathrm{H}_{1}\right)$ Os fatores produzem efeitos significativos.

Então:

Se o valor de $\rho<0,05$, rejeita-se $\mathrm{H}_{\circ}$;

Se o valor de $\rho>0,05$, aceita-se $H_{0}$.

Com base nas hipóteses formuladas e nos valores obtidos de $\alpha$, pode-se concluir que somente os fatores velocidade de alimentação (Va) e velocidade de soldagem (Vs) produziram efeitos significativos na relação Ar/Af. Essa afirmação pode ser confirmada por meio do gráfico de Pareto, Figura 2.

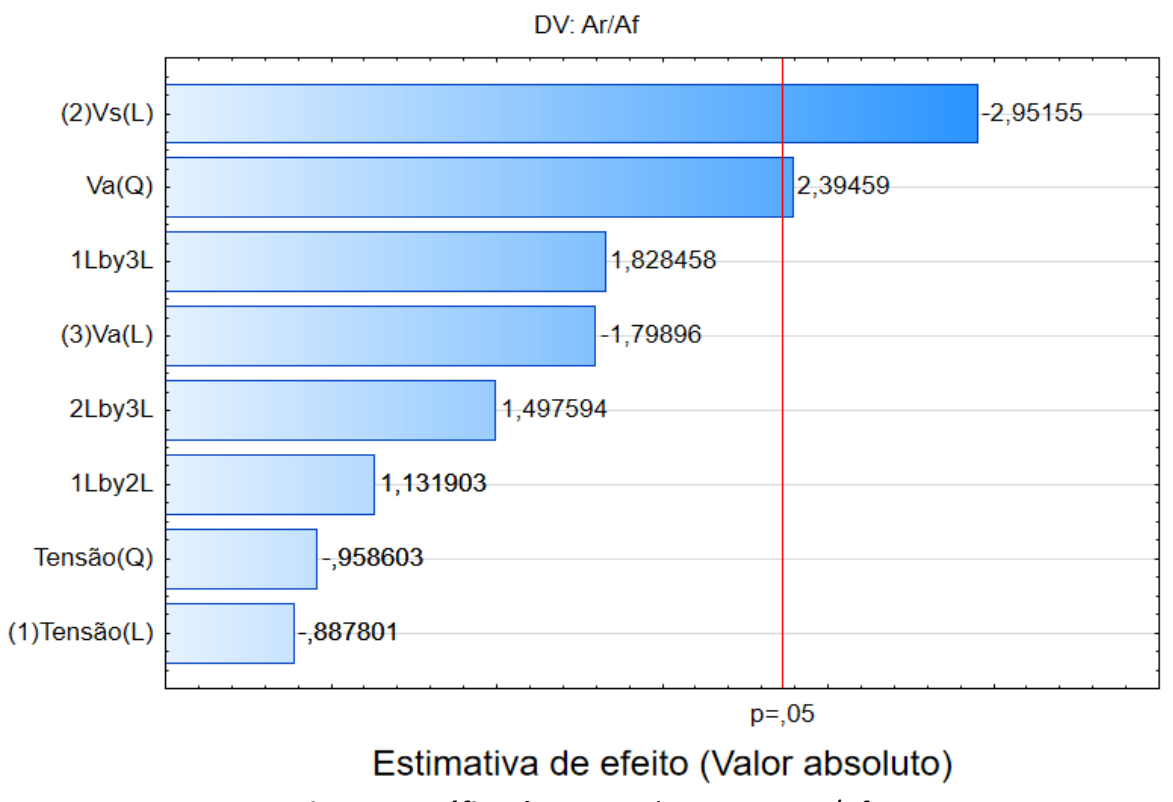

Figura 2. Gráfico de Pareto da resposta Ar/Af.

A partir do gráfico de pareto para a resposta Ar/Af, pode-se observar os fatores que são estatisticamente significativos. Assim o modelo estatístico para a resposta Ar/Af é dado pela Equação 3.

$\mathrm{Ar} / \mathrm{Af}=2,319091+0,567727 *(\mathrm{Va})^{2}-0,379000 *(\mathrm{Vs})$

Os resultados da análise de variância (ANOVA) da resposta Ar/Af, Tabela 4, revelam que a Equação 3 apresenta razoável ajuste $(R 2>0,78)$ e boa representavidade pela análise de resíduo, valor aproximado $(0,81)$ foi observado por Fratari et al. [9]. Entretanto, observa-se que o modelo não pode ser empregado para fins preditivos, haja vista ser a percentagem de variação nele explicitada, 78,38\%, um valor alto, mas muito menos interessante que os $95 \%$ desejados do modelo. Na Figura $3 a$ pode-se 
observar que os resultados das 16 relações Ar/Af apresentam uma boa concordância com a reta ajustada. O gráfico dos resíduos, Figura 3b, revela que os mesmos se distribuem de forma aleatória. Para Barros et al. [17], essas diferenças entre os valores observados e os previstos são obtidas sempre que for empregado um modelo com um número de parâmetros inferior ao número total de observações.

Tabela 4. Análise de variância (ANOVA) para a resposta relação Ar/Af.

\begin{tabular}{cccc}
\hline Fator & SQ & Gl & MQ \\
Regressão & 4,183056 & 8 & 0,522882 \\
Resíduo & 1,154188 & 7 & 0,1654884 \\
Total & 5,337244 & 15 & \\
$\mathrm{R}^{2}$ & $78,38 \%$ & & \\
\hline
\end{tabular}

Onde: $\mathrm{SQ}=$ Soma dos quadrados; $\mathrm{GI}$ = Grau de liberdade; $\mathrm{MQ}=$ Média dos quadrados.
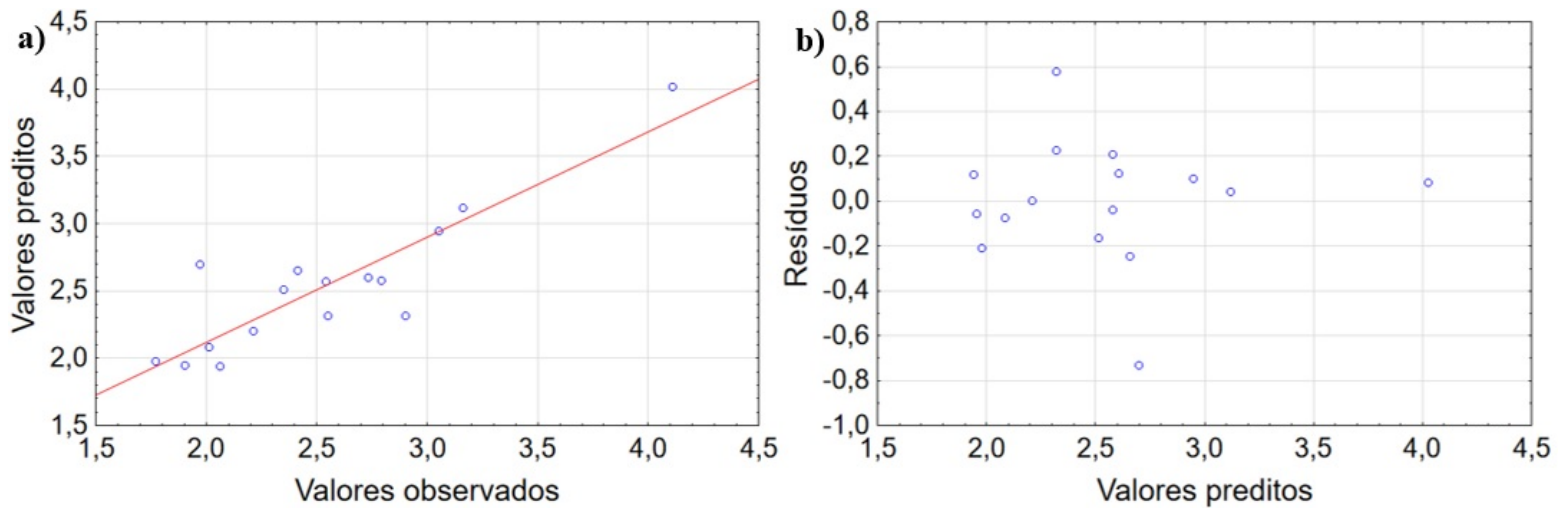

Figura 3. Ajuste do modelo para a resposta Ar/Af. (a) Valores observados $\times$ valores preditos; (b) distribuição de resíduos.

De acordo com a Equação 3, com aumento da velocidade de alimentação (Va) a relação Ar/Af também aumenta. Essa observação faz sentido, haja vista que há um aumento na quantidade de material depositado. Dessa maneira, mesmo com o valor maior da corrente devido ao aumento da Va, uma parte do calor que seria fornecida para fundir a chapa é direcionada para fundir essa maior quantidade da massa de material adicionada (aumenta Ar e diminui Af). Esses resultados são coerentes com os obtidos por Fratari et al. [9]. Com relação a velocidade de soldagem (Vs), o incremento em seus valores resulta na redução da área fundida devido a redução da energia de soldagem, além de reduzir a massa de metal depositada na composição do cordão, por consequência, há um decréscimo na relação Ar/Af.

A Figura 4 apresenta a interação entre Vs e Va, com efeito na relação Ar/Af. Por esta Figura, se observam os menores valores de Ar/Af, em torno de 2, para os valores mais elevados de velocidade de soldagem mantendo-se a velocidade de alimentação constante. Nota-se que a máxima relação da resposta Ar/Af, ocorre para valores de velocidade de soldagem por volta de $30 \mathrm{~cm} \cdot \mathrm{mim}^{-1}$ e velocidade de alimentação $10 \mathrm{~m} \cdot \mathrm{min}^{-1}$. 


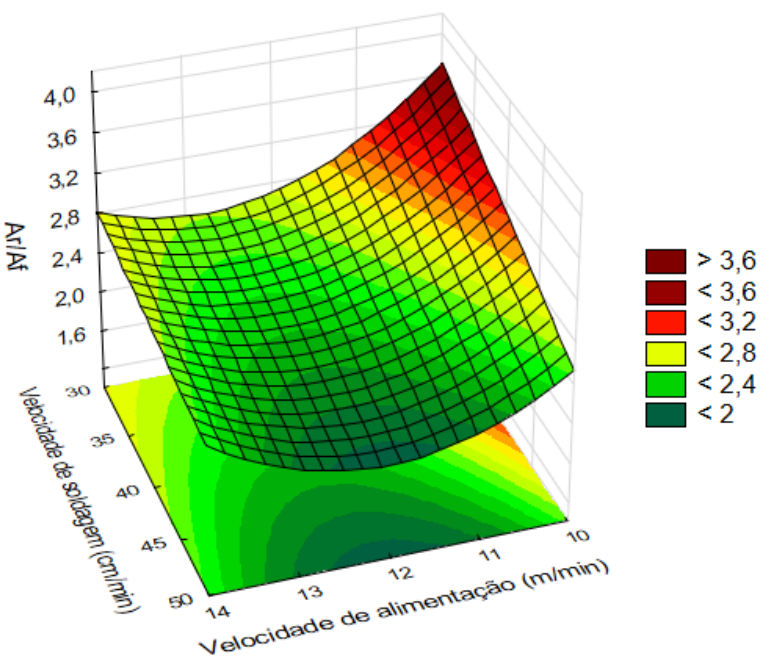

Figura 4. Superfície de resposta para relação Ar/Af sob a influência da Velocidade de soldagem versus Velocidade de alimentação, tensão 30 V.

A partir das hipóteses formuladas e dos valores de $\alpha$, o modelo estatístico para a resposta R/L é dado pela Equação 4 . No gráfico de pareto pode-se observar os fatores que são estatisticamente significativos na saída R/L, Figura 5.

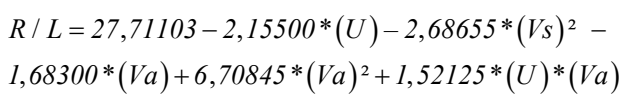

DV: R/L

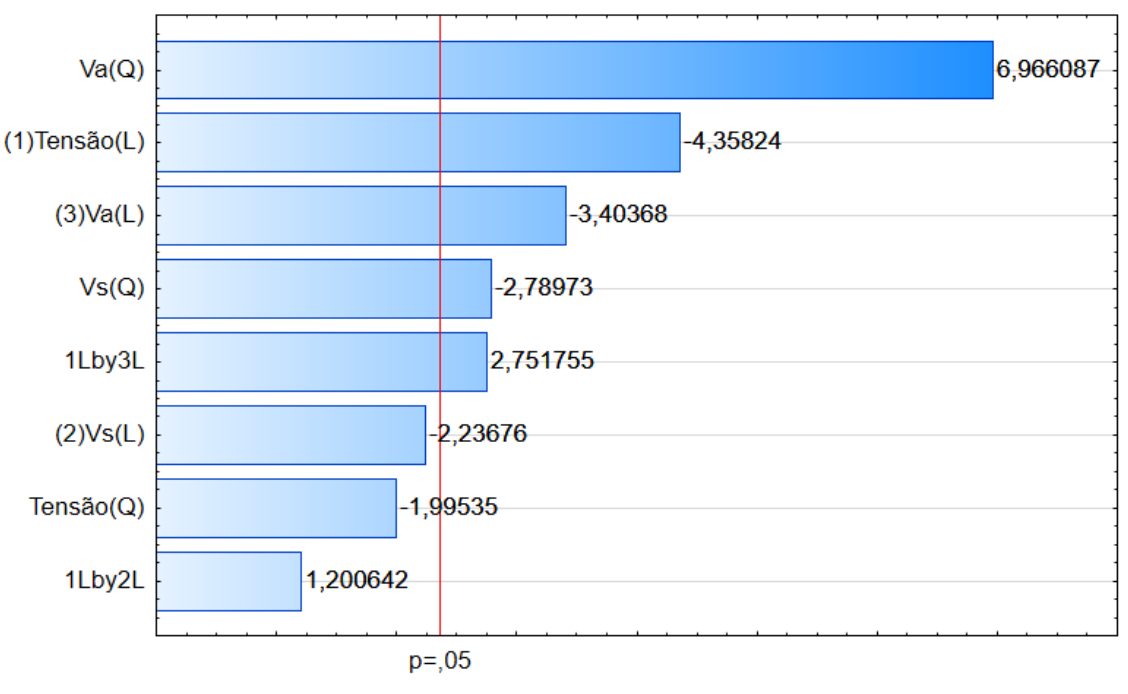

Estimativa de efeito (Valor absoluto)

Figura 5. Gráfico de Pareto da resposta R/L.

Na Tabela 5, pode-se observar o resultado da análise de variância para a resposta $\mathrm{R} / \mathrm{L}$. O valor de $\mathrm{R}^{2}$ revela que $93,0 \%$ da variação total em torno da média é explicada pela regressão. Para os resíduos ficam apenas $7,0 \%$. A soma quadrática residual abrange a parte da flutuação das respostas em torno da média que o modelo não consegue reproduzir [17]. Na Figura 6a pode-se observar que as respostas R/L apresentam boa concordância com a reta ajustada. Entretanto, ao se observar o gráfico dos resíduos, Figura $6 b$, percebe-se que ao invés de se distribuírem de forma aleatória, os resíduos negativos se concentram na região central do gráfico, e nas extremidades apenas resíduos positivos são observados. Esse comportamento pode ser resultado da necessidade de um modelo de ordem superior (cúbico), que poderia levar a uma melhor análise da variância, ajuste do modelo, além de levar a redução e a distribuição aleatória dos resíduos [17,21]. 
Tabela 5. Resultados da ANOVA para relação R/L.

\begin{tabular}{cccc}
\hline Fator & SQ & Gl & MQ \\
Regressão & 228,5821 & 8 & 28,572763 \\
Resíduo & 17,1147 & 7 & 2,444957 \\
Total & 245,6968 & 15 & \\
$\mathrm{R}^{2}$ & $93,00 \%$ & & \\
\hline
\end{tabular}

Onde: $\mathrm{SQ}=$ Soma dos quadrados; $\mathrm{GI}=$ Grau de liberdade; $\mathrm{MQ}=$ Média dos quadrados

A Equação 4 para a relação R/L revelou que os valores observados apresentam uma boa concordância com os valores preditos, Figura 6a. O esperado é que com um aumento na velocidade de alimentação, o reforço e a largura aumentem, haja vista que há um aumento na quantidade de material depositado por unidade de comprimento da solda. Entretanto, ao se manterem constantes a tensão e a velocidade de soldagem, em um determinado ponto o aumento do reforço irá se sobrepor a elevação da largura devido a redução do comprimento de arco, aumentando assim a convexidade do cordão de solda, ou seja, aumentando a relação R/L. Ao elevar a tensão há um aumento no comprimento de arco, e assim ocorre também um consequente aumento na área do arco elétrico o qual promove um maior espalhamento da poça de fusão, com o aumento da largura do cordão e redução do reforço, reduzindo assim R/L. A velocidade de soldagem apresenta um comportamento similar a tensão, ou seja, o aumentar da Vs diminui a energia de soldagem e a área fundida (menor volume da poça). Dessa maneira há uma maior conexão arco-peça aquecendo melhor as laterais do cordão devido a uma maior molhabilidade, levando a uma maior largura e menor reforço.
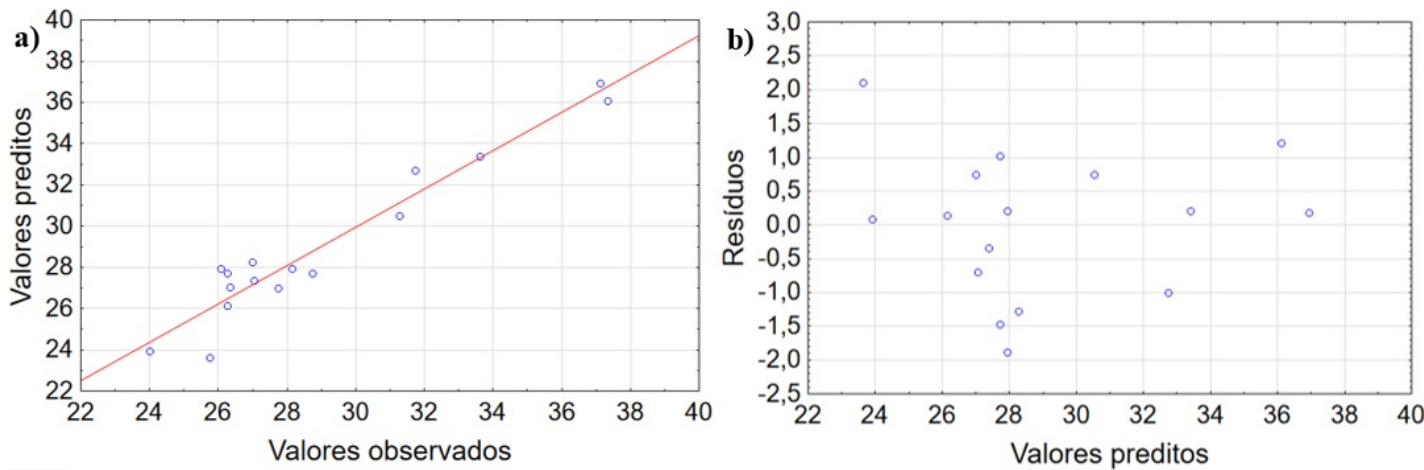

Figura 6. Ajuste do modelo para a resposta R/L. (a) Valores observados x valores preditos; (b) distribuição de resíduos.

A Figura 7 apresenta os efeitos da velocidade de alimentação e da tensão sobre a relação R/L. Fixando-se a velocidade de soldagem em $40 \mathrm{~cm} \cdot \mathrm{mim}^{-1}$, nota-se que valores baixos da resposta (R/L) são obtidos com valores de velocidade de alimentação $12,5 \mathrm{~m} \cdot \mathrm{min}^{-1}$ e tensão superior $32 \mathrm{~V}$, produzindo um cordão com baixa convexidade, pois valores próximos a $30 \%$ representam condições desejáveis para a relação $\mathrm{R} / \mathrm{L}[9,10,20]$.

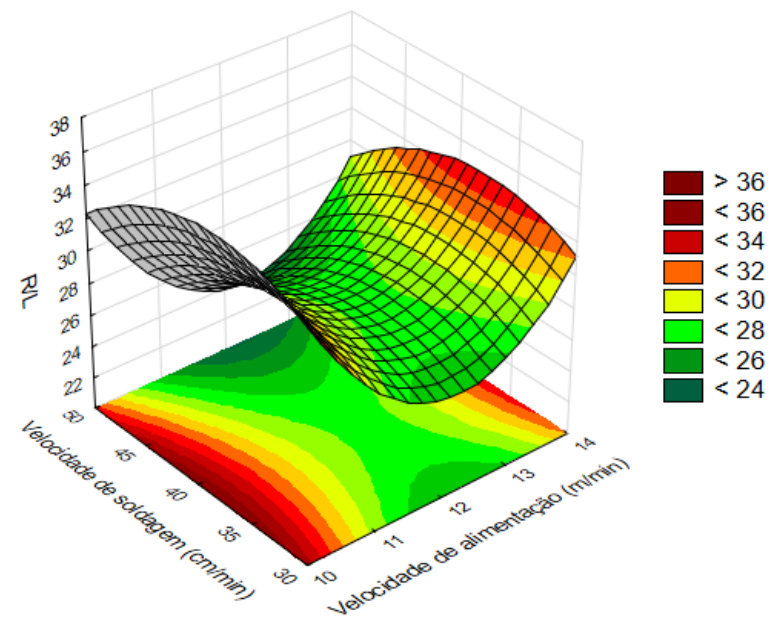

Figura 7. Superfície de resposta para relação R/L sob a influência da tensão versus Velocidade de alimentação, Velocidade de soldagem $40 \mathrm{~cm} / \mathrm{min}$. 
A estimativa das condições ótimas aplicadas à soldagem de revestimento foi realizada utilizando o método de otimização de resposta múltiplas (desirability). Neste trabalho, procurou-se maximizar relação Ar/Af buscando um incremento na produção, mas respeitando a restrição da relação $\mathrm{R} / \mathrm{L}$ menor que $30 \%$ para evitar cordões com alta convexidade.

A Tabela 6 resume a programação estabelecida no software Statistica para a entrada dos valores numéricos necessários à otimização do processo de soldagem. São especificados os valores numéricos limites e expoentes que determinam a importância da função para encontrar um valor médio desejado.

Tabela 6. Parâmetros utilizados na otimização das respostas Ar/Af e R/L.

\begin{tabular}{ccccccccc}
\hline Respostas & LI & d & M & d & LS & d & s & t \\
Ar/Af & 1,77 & 0 & 2,5 & 1 & 4,11 & 1 & 10 & 10 \\
R/L & 24,00 & 1 & 30,00 & 1 & 37,32 & 0 & 10 & 10 \\
\hline
\end{tabular}

Onde: $\mathrm{LI}$ = Limite inferior; $\mathrm{LS}$ = Limite superior; $\mathrm{d}$ = desejabilidade; onde valor será igual a 1 ser a resposta coincidir com valor alvo e 0 quando o valor encontrado se afastar da resposta desejada; $M$ = Valor alvo; s e t são expoentes da taxa de variação da desejabilidade.

A Figura 8 apresenta os resultados do algoritmo de otimização. Foi usada uma grade com 5 pontos em cada um dos três fatores, o que significa que os valores das respostas e suas correspondentes desejabilidades foram calculados em $5 \times 5 \times 5=125$ combinações de níveis de fatores. Os dois gráficos da última coluna apresentam os perfis das desejabilidades das duas respostas, definidos de acordo com os valores escolhidos para os limites e os expoentes na Tabela 6. Pode-se observar que para a relação $\mathrm{R} / \mathrm{L}$, temos um patamar de desejabilidade 1 abaixo de 30\%, já para a relação Ar/Af tem-se um patamar de desejabilidade 1 acima de 2,5. As linhas verticais (vermelhas), dos gráficos da terceira linha, sinalizam as condições de máxima desejabilidade global, que neste estudo chegou a 1 , e é obtida com $\mathrm{U}=32 \mathrm{~V}, \mathrm{Va}=14 \mathrm{~m} / \mathrm{min}$ e $\mathrm{Vs}=50 \mathrm{~cm} / \mathrm{min}$.

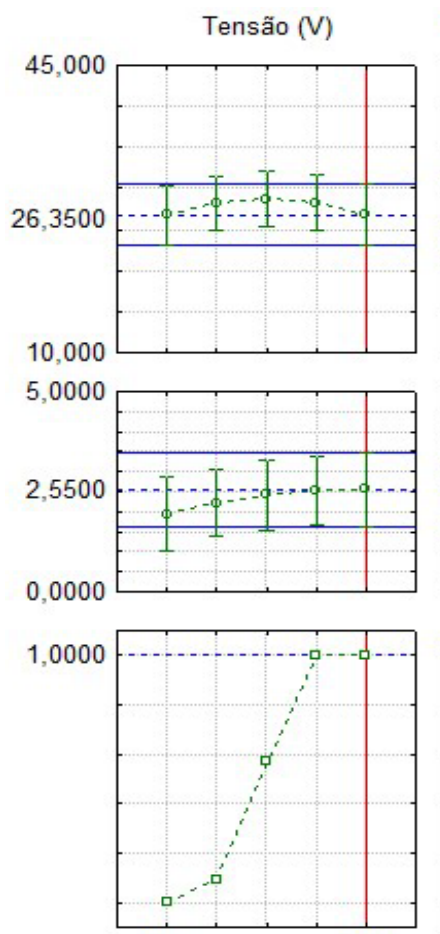

28 ,
Vel. Alimentação $(\mathrm{m} / \mathrm{min})$
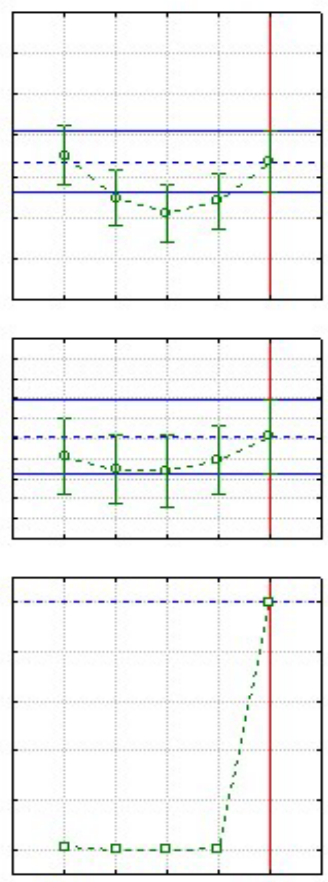

10 ,
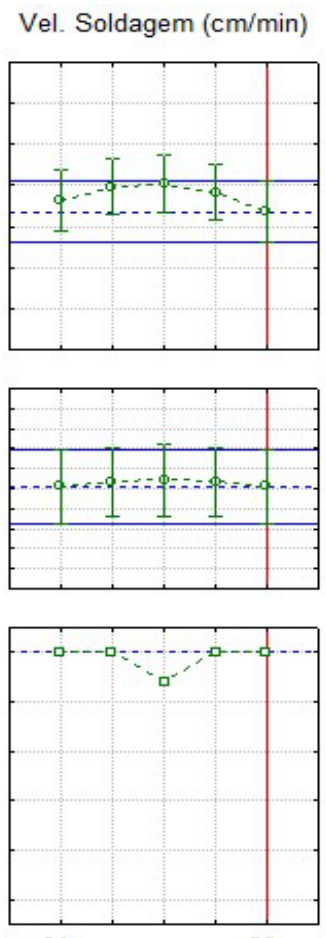

30
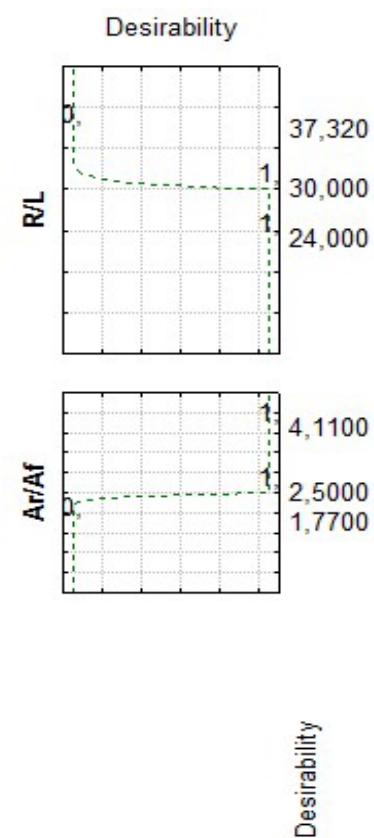

Figura 8. Gráfico da função desirability para as respostas R/L e Ar/Af.

O cordão de solda obtido através da otimização satisfaz as restrições estabelecidas nesta pesquisa. Os resultados apresentam uma relação $R / L=26,35 \%$, que satisfaz a restrição máxima de $30 \%$ estabelecida para evitar uma convexidade excessiva do cordão, e uma relação $\mathrm{Ar} / \mathrm{Af}=2,55$ que também satisfaz a condição inicial de restrição mínima de 2 , para garantir uma alta produção do cordão de solda. Portanto, na medida em que os valores se mostram condizentes com os valores estabelecidos nas restrições, eles validam os resultados obtidos para a otimização do processo. A Figura 9 apresenta o aspecto superficial e a seção transversal do cordão otimizado, que corresponde ao teste 8 dos ensaios experimentais mostrados na Tabela 3. 

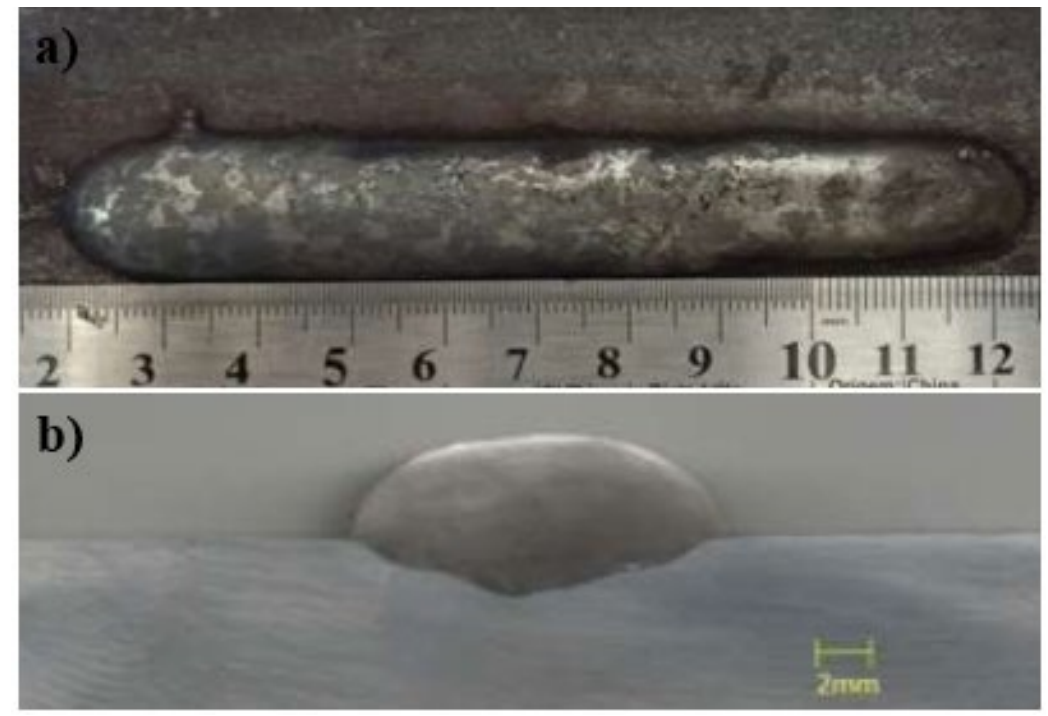

Figura 9. Cordão de solda otimizado. (a) aspecto superficial; (b) seção transversal.

\section{Conclusões}

As análises dos resultados obtidos, a partir dos procedimentos experimentais utilizados neste trabalho tornaram possível chegar as seguintes conclusões:

a) Os modelos de otimização desenvolvidos revelaram a significância estatística de Vs e Va para relação Ar/Af. Já para o modelo R/L, a resposta foi dependente dos três fatores de entrada, Va, Vs e U, além da interação $U \times$ Va;

b) A variável velo: dade de soldagem (Vs) quando aumentada, reduziu a relação Ar/Af para uma mesma velocidade de alimentação (Va). Já a superfície de resposta do modelo $\mathrm{R} / \mathrm{L}$, revelou que Va acima de $12 \mathrm{~m} / \mathrm{min}$ aumentou de forma significativa o valor da relação $\mathrm{R} / \mathrm{L}$;

c) O método desirability forneceu como resposta global um cordão de solda otimizado, com a combinação dos parâmetros: $\mathrm{U}=32 \mathrm{~V} ; \mathrm{Va}=14 \mathrm{~m} \cdot \mathrm{min}^{-1}$ e $\mathrm{Vs}=50 \mathrm{~cm} \cdot \mathrm{min}^{-1}$. Nesta condição foram obtidas as seguintes respostas: Ar/Af = 2,55; $R / L=26,35 \%$. Essa otimização resultou em características geométricas desejáveis para solda de revestimento com largura em torno de $14,53 \mathrm{~mm}$, reforço em torno de $3,83 \mathrm{~mm}$ e com diluição de $28 \%$.

\section{Agradecimentos}

Os autores agradecem ao Grupo de Estudo em Tecnologias e Pesquisa em Soldagem (GETPSOLDA) da Faculdade de Engenharia Mecânica, Belém, da Universidade Federal do Pará, Brasil, pela infraestrutura laboratorial e suporte técnico.

\section{Referências}

[1] Buchanan VE, Shipway PH, Mccartney DG. Microstructure and abrasive wear behaviour of shielded metal Arc welding Hardfacings used in the sugarcane industry. Wear. 2007;263(1-6):99-110. http://dx.doi.org/10.1016/j.wear.2006.12.053.

[2] Scotti A, Rosa LAA. Influence of oscillation parameters on crack formation in automatic Fe-B hardfacing. Journal of Materials Processing Technology. 1997;65(1-3):272-280. http://dx.doi.org/10.1016/S0924-0136(96)02415-6.

[3] Lima AC, Ferraresi VA, Reis RP. Performance analysis of weld hardfacings used in the sugar/alcohol industry. Journal of Materials Engineering and Performance. 2014;23(5):1823-1833. http://dx.doi.org/10.1007/s11665-014-0948-1.

[4] Garcia DBC. Aplicação de revestimento duro utilizando processo arame tubular com e sem adição de arame não energizado para diferentes tipos de consumíveis [dissertação de mestrado]. Uberlândia: Faculdade de Engenharia Mecânica, Universidade Federal de Uberlândia; 2011.

[5] Souza DDBG, Ferraresi VA. Aplicação de revestimento duro utilizando o processo FCAW duplo arame para diferentes tipos de consumíveis utilizados na indústria sucroalcooleira. Matéria. 2017;22(1). http://dx.doi.org/10.1590/s1517-707620170001.0126.

[6] Correa EO, Alcântara NG, Valeriano LC, Barbedo ND, Chaves RR. The effect of microstructure on abrasive wear of a Fe-Cr-C-Nb hardfacing alloy deposited by the open arc welding process. Surface and Coatings Technology. 2015;276:479-484.

http://dx.doi.org/10.1016/j.surfcoat.2015.06.026. 
[7] Wang XH, Han F, Qu S, Zou ZD. Microstructure of the Fe-based hardfacing layers reinforced by TiC-VC-Mo $2 \mathrm{C}$ particles. Surface and Coatings Technology. 2008;202(8):1502-1509. http://dx.doi.org/10.1016/j.surfcoat.2007.07.002.

[8] Lima AC, Ferraresi VA. Análise da resistência ao desgaste de revestimento duro aplicado por soldagem em facas picadoras de cana-deaçúcar. Soldagem e Inspeção. 2010;25(02):94-102. http://dx.doi.org/10.1590/S0104-92242010000200003.

[9] Fratari RQ, Schvartzman MMAM, Scotti A. Otimização dos parâmetros de tecimento para confecção de amanteigamento em chapas de aço ao carbono pelo processo TIG com arame AWS ER309L. Soldagem e Inspeção. 2010;15(3):209-217. http://dx.doi.org/10.1590/S0104-92242010000300007.

[10] Cruz LPS Jr, Ferraresi VA, Braga EM. Parâmetros otimizados para revestimento de chapas de aço carbono pelo processo MIG-DWOA com arame ER 308LSI. In: Anais do XXXV Congresso Nacional de Soldagem (CONSOLDA); 2009 Out 26-29, Piracicaba, SP. Moema: ABS; 2009.

[11] Kejelin NZ. Soldagem de revestimento de aços comuns C-Mn com superliga a base de níquel Inconel 625 [tese de doutorado]. Florianópolis: Universidade Federal de Santa Catarina; 2012.

[12] Pereira AS, Buschinelli AJA, Kejelin NZ. Avaliação da perfuração na soldagem em operação pelo processo MIG/MAG de dutos de alta resistência e baixa espessura. Soldagem e Inspeção. 2013;18(03):235-244. http://dx.doi.org/10.1590/S0104-92242013000300006.

[13] Silva MM, Batista VR, Maciel TM, Santos MA, Brasileiro TL. Otimização de parâmetros do processo de soldagem arco submerso para revestimentos anticorrosivos. Soldagem e Inspeção. 2016;21(1):115-124. http://dx.doi.org/10.1590/0104-9224/SI2101.11.

[14] Souza MTS, Fernandes MM, Miranda RCS. Otimização dos parâmetros de soldagem a ponto por resistência em uma indústria automotiva por meio da análise de experimentos e metodologia de superfície de resposta. Ciência \& Engenharia. 2013;22(2):131-140. http://dx.doi.org/10.14393/19834071.2013.20995.

[15] Rowlands H, Antony J. Application of design of experiments to a spot welding process. Assembly Automation. 2003;23(3):273-279. http://dx.doi.org/10.1108/01445150310486549.

[16] Dowlatshahi S. An application of design of experiments for optimization of plastic injection molding processes. Journal of Manufacturing Technology Management. 2004;15(6):445-454. http://dx.doi.org/10.1108/17410380410547852.

[17] Barros B No, Scarminio IS, Bruns RE. Como fazer experimentos. 4. ed. São Paulo: The Bookman. 2010. p. 102-107.

[18] KESTRA. Barras e perfis: catálogo. 2020 [acesso em 13 maio 2020]. Disponível em: http://www.kestra.com.br/uploads/product/files/825/DSAT_-_0251_-_KST_TUB_HCR_65_P_OA.pdf

[19] GERDAU. Barras e perfis: catálogo. 2020 [acesso em 13 maio 2020]. Disponível em: https://www.gerdau.com/br/pt/productsservices/products/Document\%20Gallery/catalogo-barras-e-perfis.pdf

[20] Mota CAM, Nascimento AS, Garcia DN, Silva DAS, Teixeira FR, Ferraresi VA. Revestimento de níquel depositado pela soldagem MIG e MIG com arame frio. Soldagem e Inspeção. 2017;21(4):483-496. http://dx.doi.org/10.1590/0104-9224/si2104.08.

[21] Rodrigues MI, Lemma AF. Planejamento de experimentos e otimização de processos. Campinas: AMIC; 2009. 